\title{
Crecimiento y ciclo reproductivo de Polymesoda radiata (Bivalvia: Corbiculidae) en Costa Rica
}

\author{
Eleazar Ruiz Campos ${ }^{1}$, Jorge Cabrera Peña², Rafael A. Cruz ${ }^{2}$ y José A. Palacios² \\ 1 Centr de investigaciones en Ciencias del Mar (CIMAR), Universidad de Costa Rica, 2060 San José, Costa Rica. \\ 2 Escuela de Ciencias Biológicas, Universidad Nacional, Heredia 86-3000, Costa Rica.
}

\section{Recibido 23-IX-1997. Corregido 18-VI-1998. Aceptado 23-VI-1998.}

\begin{abstract}
A population of green clam, Polymesoda radiata was sampled from August 1992 through July 1993 in Bebedero river, Guanacaste, Costa Rica $(n=978)$ for demographic data. Mean values (followed by ranges) were: shell length $32.29 \pm 7.63 \mathrm{~mm}(6.9-44.10 \mathrm{~mm})$. The mean total weight was $11.13 \pm 5.71 \mathrm{~g}(0.4-23.98 \mathrm{~g})$. Data analysis showed a isometric relationship between length and total weight, represented by the equation : $\mathrm{Pt}=2.07 \times 10^{-4} \mathrm{Lt}^{3.01}(r=0.987$,

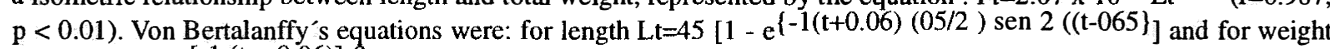
$\mathrm{Pt}=26.53\left(1-\mathrm{e}^{[-1(\mathrm{t}+0.06)]}\right)^{3}$. The growth rate for total length was $2.25 \pm 0.66 \mathrm{~mm} / \mathrm{month}$ for the first year $(0.83 \pm$ 0.24 the second year and $0.26 \pm 0.1$ for other months). Individuals become sexually mature throughout the year. The mean sex ratio was 1.00 male : 0.61 females. Spawning suggested that minimum size at sexual maturity was $7.80 \mathrm{~mm}$ of Lt. The largest number of ripe individuals was found in the dry season. Spawning peaks were in December and March. Histologically this is a dioecious bivalve with stable gonocorism.
\end{abstract}

Key words: Polimesoda radiata, bivalvia, growth, gonadic development, spawning cycle, clam, Costa Rica.

Polymesoda radiata (Hanley 1844) conocida comúnmente como almeja verde o almeja de lodo, se distribuye desde Mazatlán, México hasta Ecuador; se encuentra principalmente cerca de la desembocadura de los ríos en la zona de transición entre agua salobre y dulce (Morton 1985, Severey 1993).

La información de que se dispone sobre $P$. radiata en Centroamérica, en especial Costa Rica es escasa, se refiere principalmente a trabajos de tipo taxonómico (Olsson 1961, Keen 1971, Bernard 1983, Severey 1993, Cruz y Jiménez 1994), a pesar de ser una especie importante para consumo humano.

Entre los principales trabajos realizados en el género Polymesoda se encuentran los de
Castaing et al. (1980), quienes estudiaron la distribución de $P$. inflata en los manglares de la costa pacífica de Costa Rica; Duobinis-Gray y Hackney (1982), informan de la distribución espacial y estacional de $P$. caroliana en Mississippi, EUA; Báez (1985), estudió el ciclo reproductivo y la composición química de la carne de $P$. inflata en Pochote, Costa Rica; y Morton (1985) informa de la e strategia reproductiva de P. erosa en Hong Kong, China.

El objetivo de este trabajo fue determinar la biometría, crecimiento y ciclo reproductivo de $P$. radiata en el río Bebedero, Guanacaste, Costa Rica. 


\section{MATERIALES Y MÉTODOS}

Se trabajó con un total de 978 ejemplares de Polymesoda radiata, recolectados mensualmente en forma manual y al azar entre agosto de 1992 y julio de 1993, en un banco natural en la ribera izquierda del Río Bebedero a $5 \mathrm{~km}$ de Puerto Níspero, Costa Rica ( $10^{\circ} 16^{\prime} 44^{\prime \prime} \mathrm{N}$ y $85^{\circ}$ 14'37' O).

A cada ejemplar se le midió longitud total $(\mathrm{Lt})$, altura (A) y diámetro (D) con una precisión de $0.01 \mathrm{~mm}$ y el peso total (Pt) con una precisión de $0.05 \mathrm{~g}$ (Cruz 1989, Caballero et al. 1996/1997). Se determinó la estadística descriptiva, matriz de correlación entre las variables medidas, el crecimiento relativo $\mathrm{Pt}=\mathrm{a} \mathrm{Lt}^{\mathrm{b}}$ (Theisen 1968) y las ecuaciones de crecimiento de Von Bertalanffy (Gayanilo et al. 1989).

Una muestra de 186 ejemplares se utilizó para establecer el ciclo reproductivo y la proporción sexual y otra de 55 individuos para determinar la talla mínima reproductiva; éstos se agruparon arbitrariamente en cinco ámbitos de Lt : 1 (3.60-5.60 mm), $2(5.61-7.61 \mathrm{~mm}), 3$ (7.62-9.62 mm), 4 (9.63-11.63 mm), 5 (11.64$14.64 \mathrm{~mm}$ ). Se trabajó con secciones de la masa visceral y del manto, las que fueron fijadas en Bouin, posteriomente incluidas en parafina $y$ cortadas a 7 micras para su tinción con hematoxilina-eosina (Howard y Smith 1983). El examen al microscopio del tejido gonadal fue realizado asignando las categorías de inmaduro o indiferenciado, maduro y desovado (machos y hembras), para la talla a primera madurez sexual. Para el estudio histológico del ciclo reproductivo de los adultos ( $>$ de $7.80 \mathrm{~mm}$ de $\mathrm{Lt}$ ) se asignaron las categorías de gametogénesis, maduros y desovados de acuerdo con Cruz (1984 a, b), Palacios (1985) y Cruz y Villalobos (1993). Las mismas se definen como sigue:

Gametogénesis: Fase que se caracteriza por la multiplicación y ordenamiento de las células sexuales. En las hembras los oogonios se pueden encontrar en la periferia unidos a las paredes de la gónada y también se encuentran óvulos periformes. El número de óvulos libres y adheridos es aproximadamente igual. En los machos las espermátidas pueden estar pegadas a la periferia de los lobulillos o encontrarse sin ningún ordenamiento.

Maduros: En las hembras los óvulos se ven libres en el lumen y de forma redondeada llenando todo el espacio. Los lobulillos se consideran maduros cuando el número de óvulos libres es mayor que los adheridos. Los machos muestran los lobulillos totalmente llenos de espermatozoides con los flagelos dirigidos hacia el centro del lumen y formando bandas concéntricas.

Desovados: En las hembras los lóbulos se ven vacíos con pocos óvulos redondeados. En los machos se ven pocos espermatozoides hacia el centro del lumen. En ambos casos las paredes de las gónadas se comienzan a desintegrar.

\section{RESULTADOS}

La estadística descriptiva para $P$. radiata muestra que la mayor desviación estándar se presenta con el peso total y que el mayor ámbito se obtiene para Lt (Cuadro 1). La variable que mejor se ajusta para la descripción taxonómica es altura, dada su poca variabilidad (coeficiente de variación=22.74). Los coeficientes de correlación para las variables morfológicas

CUADRO 1

Estadística descriptiva para Polymesoda radiata en río Bebedero, Guanacaste, Costa Rica.

$\begin{array}{cccccc}\text { Parámetro } & \text { Promedio } & \text { DE } & \text { Mínimo } & \text { Máximo } & \text { Coef. Var. } \\ \text { Longitud total (mm) } & 32.29 & 7.63 & 6.90 & 44.10 & 23.63 \\ \text { Altura (mm) } & 28.45 & 6.47 & 6.80 & 40.50 & 22.74 \\ \text { Diámetro (mm) } & 17.99 & 4.18 & 4.30 & 32.20 & 23.23 \\ \text { Peso total }(\mathrm{g}) & 11.13 & 5.71 & 0.40 & 23.98 & 51.30\end{array}$


presentan un ámbito entre 0.927 y 0.9888 , o sea, explican satisfactoriamente las variables dependientes, dado que los valores son significativamente altos a un valor crítico de \pm $0.56108(\mathrm{p}<0.05)$. $\mathrm{mm}$ de Lt se distinguen células sexuales tanto en machos como en hembras, sin embargo, es solo a partir de la talla $7.80 \mathrm{~mm}$ de Lt que se encuentran individuos desovados.

\section{CUADRO 2}

Frecuencia de los estadios de madurez sexual para establecer la talla a la primera madurez sexual en Polymesoda radiata ( $n$ = 55), río Bebedero, Costa Rica.

$\begin{array}{ccccc}\text { Tallas }(\mathrm{mm}) & \begin{array}{c}\text { Fases de Madurez Gonádica } \\ \text { Indefinidos } \\ \%\end{array} & \begin{array}{c}\text { Desovados } \\ \%\end{array} & \begin{array}{c}\text { Machos } \\ \%\end{array} & \begin{array}{c}\text { Hembras } \\ \%\end{array} \\ 3.60-5.60 & 100.0 & - & - & - \\ 5.61-7.61 & - & - & 66.7 & 33.3 \\ 7.62-9.62 & - & 33.3 & 25.0 & 41.7 \\ 9.63-11.63 & - & 5.6 & 77.7 & 16.7 \\ 12.64-14.64 & - & - & 58.0 & 42.0\end{array}$

La relación Lt-Pt para la población total se rige por la ecuación $\mathrm{Pt}=2.07 \times 10^{-4} \mathrm{Lt}^{3.01}$ con un coeficiente de correlación ( $r$ ) de 0.987 ( $p<0.01$ ), lo que indica un crecimiento de tipo isométrico.

El crecimiento calculado según la ecuación de Von Bertalanffy se rige por las ecuaciones : $\mathrm{Lt}=45\left[1-\mathrm{e}^{\{-1(\mathrm{t}+0.06)(05 / 2) \operatorname{sen} 2(\mathrm{t}-065\}}\right] \mathrm{pa}-$ ra longitud total y $\mathrm{Pt}=26.53\left(1-\mathrm{e}^{[-1(\mathrm{t}+0.06)]}\right)^{3}$ para peso total. Se estima que $P$. radiata tiene una tasa de crecimiento en $\mathrm{Lt}$ de $2.25 \pm 0.66$ $\mathrm{mm} / \mathrm{mes}$ para el primer año, $0.83 \pm 0.24 \mathrm{~mm}$ imes para el segundo año y a partir de los 25 meses es $0.26 \pm 0.10 \mathrm{~mm} / \mathrm{mes}$. La tasa de crecimiento en Pt es de $0.61 \pm 0.33 \mathrm{~g} / \mathrm{mes}$ para el primer año, de $0.87 \pm 0.11 \mathrm{~g} / \mathrm{mes}$ para el segundo año y a partir de los 25 meses en adelante 0.41 $\pm 0.14 \mathrm{~g} / \mathrm{mes}$.

Considerando el ámbito de longitudes totales encontradas (6.09 - $44.10 \mathrm{~mm}$ ) se estima que la estructura de edad para la población de $P$. radia$t a$ en el río Bebedero oscila entre 0.25 y 3.2 años.

La frecuencia de estadíos por ámbito de tallas (Cuadro 2) indica que todos los ejemplares examinados y comprendidos entre los 3.60-5.60 $\mathrm{mm}$ de Lt son indefinidos, caracterizandose por no presentar histológicamente coloración alguna en la región gonadal y sólo se observan células de tejido conjuntivo. A partir de la talla 6.62
La distribución por sexos (Fig. 1), indica que durante todo el periodo de estudio predominan los machos (8 meses) y sólo predominan las hembras en agosto y diciembre. Del total de 186 ejemplares examinados, 113 fueron machos $(60.75 \%)$ y 69 hembras $(37.10 \%)$ con una proporción sexual de 1.00 macho: 0.61 hembras. Se encontró en mayo 4 ejemplares hermafroditas, lo que representa un $2.15 \%$ del total de la población.

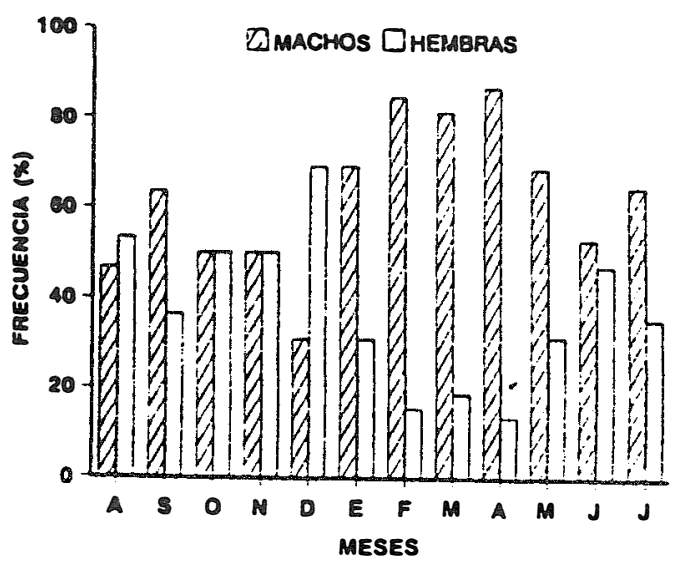

Fig. 1. Frecuencia mensual de sexos de Polymesoda radiat $a$ en el río Bebedero, Guanacaste, Costa Rica. 
La variación mensual del desarrollo gonadal de $P$. radiata (Fig.2), describe individuos en los diferentes estadíos durante todos los meses de estudio. El periodo de mayor desove ocurre desde diciembre a abril y corresponde a la época seca, con un pico de más intensidad en el mes de marzo $(92.3 \%)$ y un ámbito entre $69.2 \%$ y $92.3 \%$. El mayor porcentaje de gametogénesis ocurre en enero $(28.60 \%)$.

\section{Dgametogenesis Dmaduro Noesovado}

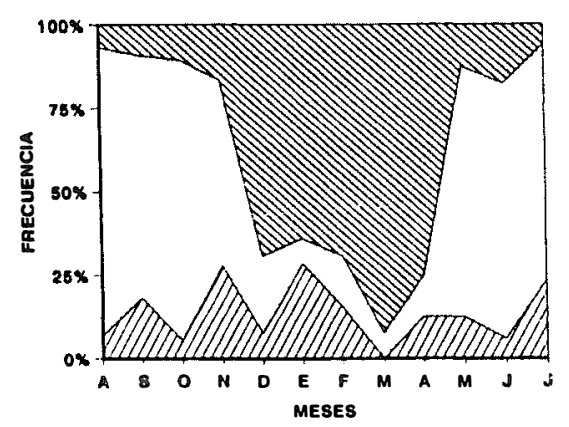

Fig. 2. Variación mensual del desarrollo gonadal de Polymesoda radiata en río Bebedero, Guanacaste, Costa Rica

\section{DISCUSION}

Los valores máximos de longitud total, altura y diámetro encontrados en el presente estudio $(44.10 \mathrm{~mm}, 40.50 \mathrm{~mm}$ y $32.20 \mathrm{~mm}$ respectivamente), son mayores que los informados para la misma especie por Olsson (1961) (39.5 mm, $36.00 \mathrm{~mm}$ y $28.0 \mathrm{~mm}$ ), Keen (1971) (40 mm, 36 mm y $28 \mathrm{~mm}$ ) y Cruz y Jiménez (1994) (43 mm de Lt y 22.5 de diámetro).

Las correlaciones entre los parámetros morfológicos medidos en esta especie, explican satisfactoriamente el comportamiento de las variables dependientes $(r<0.927$ y $\mathrm{p}<0.05$, a un valor crítico de \pm 0.56108 ) y el bajo coeficiente de variación de ellos, permite que estas variables puedan ser utilizadas en la caracterización taxonómica de la especie coincidiendo con lo informado para Polymesoda inflata por Báez (1985).
La tasa de crecimiento en Lt para el primer año $(2.25 \pm 0.66 \mathrm{~mm} / \mathrm{mes})$, es mayor que a la informada para Polymesoda carolina en Mississippi, EUA por Duobinis-Gray y Hackney (1982) (entre 0.7 y $1.4 \mathrm{~mm} / \mathrm{mes}$ ) y para Donax dentifer en Playa Garza, Costa Rica por Palacios et al. (1983)(2.07 mm/mes); siendo menor que para Donax denticulata en la India por Wade (1969) $(3.0 \mathrm{~mm} / \mathrm{mes})$.

El concepto de edad límite propuesto por Taylor (1958), sugiere que $P$. radiata alcanzaría el $95 \%$ de su Lt los 3.25 años y la longitud asintótica $(45 \mathrm{~mm})$ a los 3.33 años.

El crecimiento de esta especie es de tipo isométrico entre la longitud total y el peso total. La tasas de crecimiento en $\mathrm{Pt}$ (entre $0.87 \pm 0.11$ $\mathrm{g} / \mathrm{mes}$ y $0.41 \pm 0.14 \mathrm{~g} / \mathrm{mes})$, son menores que las informadas para Dosinia exoleta en Noruega por Tunberg (1983) (2.81 g/mes) y para Protothaca asperrima en Colorado de Abangares, Costa Rica por Palacios (1985) (4.0 g/mes).

Los resultados histológicos indican que Polymesoda radiata corresponde a una especie dioica, con un gonocorismo normal o estable dado su baja incidencia de hermafroditismo (2.15\%), lo que coincide con lo informado por Heller (1993) para diferentes moluscos; por Báez (1985) para P. inflata en Costa Rica; por Cruz (1986) para Anadara grandis en Costa Rica; y Cruz y Villalobos (1993) para Mytella guyanensis en el Golfo de Nicoya, Costa Rica.

La proporción sexual promedio encontrada en $P$. radiata (1.00 macho:0.61 hembras) es semejante a lo informado por Báez (1985) para Polymesoda inflata en el manglar de Pochote, Costa Rica (1.00:0.65) y por Palacios (1985) para Protothaca asperrima en Colorado de Abangares, Costa Rica (1.00:0.63); difiere con lo informado por Cruz (1984 c) para Anadara similis en Jicaral, Costa Rica (1.00:0.83).

$P$. radiata presenta ejemplares desovados durante todo el año, coincide con lo informado por Giese y Pearse (1974) quienes señalan que los moluscos bivalvos de las zonas tropicales se caracterizan por tener desoves prolongados en su ciclo reproductivo; por Báez (1985) para Polymesoda inflata en el manglar de Pochote, Costa Rica; para Protothaca asperrima en Colorado 
de Abangares, Costa Rica por Palacios (1985); y para Crassostrea columbensis en Golfo de Nicoya, Costa Rica por Caballero et al. (1996/1997).

En $P$. radiata el período de máximo desove ocurrió en los meses de la estación seca y por lo tanto los más calientes, coincidiendo con lo informado para Prototaca asperrima por Palacios (1985) y para Anadara grandis por Cruz (1987).

Dependiendo del tamaño de la especie, la talla mínima reproductiva se presenta a diferentes longitudes, es así que en $P$. radiata, la talla mínima para ambos sexos en que fue posible observar presencia de células sexuales fue 6.62 $\mathrm{mm}$ de Lt. Sin embargo, sólo se encontró ejemplares desovados a partir de la talla $7.80 \mathrm{~mm}$ de Lt por lo que se puede inferir que la talla mínima reproductiva corresponde a esta última y equivale a la cuarta parte de la longitud informada para la especie por Keen (1971).

La talla mínima reproductiva en $P$. radiata $(7.80 \mathrm{~mm})$ se encuentra en los ámbito reportados por Hall (1984) para Corbicula fluminea en Carolina del Norte, EUA (6.5-10.0 mm Lt); es menor que lo informado por Kennedy y Huekelem (1985) para Corbicula sp. en Maryland, EUA (9.6 mm Lt); por Morton (1985) para Polymesoda erosa en Hong Kong, China (35 mm); por Cruz (1987) para Anadara grandis en Costa Rica (21.0 mm); y por Morton (1990) para Gafrarium pectinatum en Hong Kong, China (16.0-20.0 mm).

Si se desea establecer una explotación sostenible del recurso $P$. radiata, se sugiere que su comercialización se realice con especímenes que tengan tallas superiores a los $30 \mathrm{~mm}$, la que se alcanza a los 13 meses de edad, debido a que a partir de esa talla su crecimiento mensual disminuye, lo que coincide con Vélez (1976) quien señala que los moluscos en el tropico disminuyen su tasa de crecimiento después de haber alcanzado la madurez sexual.

\section{RESUMEN}

Se determinó el crecimiento, biometría, desarrollo gonádico, ciclo reproductivo y alla mínima reproductiva en Polymesoda radiata en el río Bebede o, Guanacaste Costa Rica, entre agosto 1992 y julio 1993. El promedio de la longitud total fue $32.29 \pm 7.63 \mathrm{~mm}$ (ámbito entre 6.90 to 44.10 $\mathrm{mm}$ ). El peso total fue $11.13 \pm 5.71 \mathrm{~g}$ (ámbito entre 0.4 to $23.98 \mathrm{~g}$ ). La relación $\mathrm{Lt}-\mathrm{Pt}$ determinó que el crecimiento es de tipo isométrico y se rige por la ecuación $\mathrm{Pt}=2.07 \times 10^{-4}$ $\mathrm{Lt}^{3.01}(\mathrm{r}=0.987, \mathrm{p} 0.01)$. La ecuación generalizada de von Bertalanffy se rige para $\mathrm{Lt}$ por $\mathrm{Lt}=45\left[1-\mathrm{e}^{\{-1(\mathrm{t}+0.06)}\right.$ $(05 / 2) \operatorname{sen} 2((\mathrm{t}-065)]$ y para peso tot por $\mathrm{Pt}=26.53(1-\mathrm{e}$ $[-1(t+0.06)])^{3}$. La tasa de crecimiento para $L t$ fue de 2.25 $\pm 0.66 \mathrm{~mm} / \mathrm{mes}$ para el primer año, $0.83 \pm 0.24 \mathrm{~mm} / \mathrm{mes}$ para el segundo año y $0.26 \pm 0.1 \mathrm{~mm} / \mathrm{mes}$ para el resto de los meses. Durante todo el año se encontraron individuos en las diferentes etapas reproductivas. La proporción sexual para el periodo de estudio fue de 1.00 macho:0.61 hembras. La talla a la que se distinguen células sexuales tanto para machos como hembras fue de $6.62 \mathrm{~mm}$ y se establece como talla mínima reproductiva $7.80 \mathrm{~mm}$, ya que a partir de ésta se observaron individuos desovados. La mayor actividad de desove se observó durante la época seca con dos picos máximos de desove al año, uno en diciembre y el otro en marzo. El análisis histológico determinó que la especie es dioica con un bajo porcentaje de hermafroditismo en la población $(2.15 \%)$

\section{REFERENCIAS}

Báez, R. 1985. Evaluación de algunos aspectos de la biología del molusco.Polymesoda inflata (Philipp 1851) (Bivalvia:Corbiculidae), en el manglar de Pochote, Puntarenas, Costa Rica. Tesis de Licenciatura. Escuela de Biología, Universidad de Costa Rica, San José, Costa Rica. $70 \mathrm{p}$

Bernard, F. 1983. Catalogue of the living Bivalvia of the Eastern Pacific Ocean: Bering Strait to Cape Horn. Can. Spec. Publ. Fish. Aquat. Sci. 102 p.

Caballero, A. F., J. Cabrera \& Y. Solano. 1996/1997. Descripción del crecimiento y madurez sexual de una población de Crassostrea columbiensis (Mollusca: Bivalvia). Rev. Biol. Trop. 44/45:335-339.

Castaing, A., J. Jiménez \& C. Villalobos. 1980. Observaciones sobre la ecología de manglares de la costa Pacífica y en relación con la distribución del molusco Geloina inflata, (Philippi) (Pelecypoda: Corbiculidae). Rev. Biol. Trop. 28:323-339.

Cruz, R. 1984 a. Algunos aspectos reproductivo y variación mensual del índice de condición de Anadara tuberculosa (Bivalvia: Arcidae) en Costa Rica. Brenesia 31:21-24. 
Cruz, R. 1984 b. Algunos aspectos de la reproducción en Anadara tuberculosa (Pelecypoda:Arcidae) de Punta Morales, Puntarenas, Costa Rica. Rev. Biol. Trop. 32:45-50.

Cruz, R. A. 1984 c. Algunos aspectos de la reproductivos y variación mensual del índice de condición de Anadara similis (Pelecypoda: Arcidae) de Jicaral, Puntarenas, Costa Rica. Brenesia 22:95-105.

Cruz, R. 1986. The reproductive cycle of the mangrove cockle Anadara grandis (Bivalvia:Arcidae), in Costa Rica. Brenesia. 27/28:37-47.

Cruz, R. 1987. Tamaño y madurez sexual en Anadara grandis (Pelecypoda:Arcidae). Brenesia 27:9 -12.

Cruz, R. A. 1989. Variación mensual de la condición, composición bioquímica (proximal) y ciclo reproductivo del mejillón Mytella guyanensis (Bivalvia:Mytilidae). Tesis de Licenciatura en Biología, Escuela de Ciencias Biológicas, Universidad Nacional, Heredia, Costa Rica. $66 \mathrm{p}$.

Cruz, R. \& C. Villalobos. 1993. Shell length at sexual maturity cycle of Mytella guyanensis (Bivalvia: Mytilidae) from Costa Rica. Rev. Biol. Trop. 41:89-92.

Cruz, R. \& J. Jiménez. 1994. Moluscos asociados a las áreas de manglar de la Costa Pacífica de América Central. EFUNA, Heredia, Costa Rica. 182 p.

Duobinis-Gray, E. \& C. Hackney. 1982. Seasonal and spatial distribution of carolina marsh clam, Polymesoda carolina (Bosch) in Mississipi Tidal marsh. Estuaries 5:102-109.

Gayanilo, F. C., J. Soriano \& D. Pauly. 1989. A draf guide to Compleat ELEFAN. INCLARM software 2.70. International Center for Living Aquatic Resources Management. Manila. Contribution \# 435:70 p.

Giese, A. \& J. Pearse. 1974. Introduction general principles. In: Reproduction of marine invertebrates. A. C. Giese y J. S. Pearse (eds). Vol. I. Academic, Nueva York. 546 p.

Hall, J. 1984. Production of immature Corbicula fluminea (Bivalvia: Corbiculidae), in Lake Norman, North Carolina. Nautilus 98:153-159.

Heller, J. 1993. Hermaphroditism in molluscs. Biol. J. Linnean Soc. 48:19-42.

Howard, D. \& C. Smith. 1983. Histological techniques for marine bivalve mollusks. NOAA Tecnical Memorandum NMFS/NEC. 25:25-97.
Keen, A. 1971. Sea shell of tropical west América. Stanford University, Stanford. 1064 p.

Kennedy, V. \& L. Huekelem. 1985. Gametogenesis and larval production in a population of the introduced asiatic clam, Corbicula .sp (Bivalvia:Corbiculidae), in Maryland. Biol. Bull. 168:50-60.

Morton, B. 1985. The reproductive strategy of the mangrove bivalve Polymesoda (Geloina) erosa (Bivalvia:Corbiculidae) in Hong Kong. Malacol. Review. 18: 83-89.

Morton, B. 1990. The life cycle and sexual strategy of Gafrarium pectinatum (Bivalvia:Veneridae) in a Hong Kong mangrove. Malacol. Review 23: 53-62.

Olsson, A. 1961. Mollusks of the tropical eastern Pacific, particulary from the southern half of the PanamicPacific faunal province (Panamá to Perú). PanamicPacific Pelecypoda Paleontological Res. Inst. Ithaca, Nueva York. 574 p.

Palacios,J. A., R. A. Cruz \& O. Pacheco.1983. Estructura poblacional y cuantificación de Donax dentifer Hanley, 1843 (Pelecypoda: Donacidae) en Playa Garza, Puntarenas, Costa Rica. Rev. Biol. Trop. 31:251-255.

Palacios, J. 1985. Biometría, crecimiento y maduración sexual en la almeja blanca Protothaca asperrima (Pelecypoda:Veneridae) en Colorado de Abangares, Guanacaste, Costa Rica. Tesis de Licenciatura. Escuela de Ciencias Biológicas. Universidad Nacional, Heredia, Costa Rica. 85 p.

Severey, V. 1993. Taxonomic revision and phylogeny of the genus Polymesoda (Rafinesque 1820) (Bivalvia:Corbiculidae). Tesis Doctoral. University of Maryland, Maryland. $150 \mathrm{p}$.

Theisen, B. F. 1968. Growth and mortality of culture mussels in Danish Wadden Sea. Mddr. Danm. Fisk. 6:47-48.

Taylor, C. C. 1958. Cod growth and temperature. J. Cons. Inst. Explor. 23:366-370.

Tunberg, B. 1983. Growth of Dosinia exoleta L (Bivalvia) in Raunefjorden, Western Norway. SARSIA 68:41-45.

Vélez, A. R. 1976. Crecimiento, edad y madurez sexual del ostión Crassostrea rhizophorae de Bahía Mochima. Bol. Inst. Oceanogr. Univ. Oriente 15:65-72.

Wade, B. 1969. Studies on biology of West Indian beach clam, Donax denticulata Linneaus 2. Life history. Bull. Mar. Sci. 18:876-901. 